Proceedings of the 17th Czech and Slovak Conference on Magnetism, Košice, Slovakia, June 3-7, 2019

\title{
Modelling the Fluxgate Sensors with Magnetic Field Concentrators
}

\author{
R. SzeWCZYK ${ }^{a, *}$, A. Ostaszewska-Liżewska ${ }^{a}$ AND P. RABACK ${ }^{b}$ \\ ${ }^{a}$ Warsaw University of Technology, Faculty of Mechatronics, sw. A. Boboli 8, 02-525 Warsaw, Poland \\ ${ }^{b}$ CSC - Scientific Computing Ltd., Tekniikantie 15a D, Box 405, FIN-02101 Espoo, Finland
}

\begin{abstract}
This paper presents the efficiency analysis of magnetic flux concentrator attached to the core of fluxgate sensor made of amorphous alloy ribbon. Simulations were carried out using open-source software toolchain covering Netgen, Elmer FEM, and ParaView. The results indicate that the increase of the length of the core significantly reduces the demagnetization factor, which increases the sensitivity of fluxgate sensor. However, the use of magnetic flux concentrator does not lead to significant increase of sensitivity of fluxgate sensor with core made of thin layer magnetic material.
\end{abstract}

DOI: 10.12693/APhysPolA.137.700

PACS/topics: fluxgate sensor, amorphous alloy, finite elements modelling

\section{Introduction}

Since its invention in 1936 [1], fluxgate magnetometers are one of the most robust and sensitive magnetic sensors widely used in geology [2], archeology [3], and space research [4]. It was estimated that, until now, over two hundreds of fluxgate magnetometers [4] were used for investigation of magnetosphere of Earth, Moon, and other planets of the Solar System. On the other hand, fluxgate magnetometers have significant drawback, limiting their applications, e.g., in industrial automation. Such sensors are rather big due to the use of bulk cores made of magnetic materials. Moreover, production of fluxgate sensors is expensive, especially due to the fact that most of their elements have to be produced and assembled manually.

To avoid these drawbacks, recent developments of fluxgate sensors are focused on utilization of cores made of amorphous alloy ribbons, whereas the whole sensor can be developed within single printed board circuit [5]. Such fluxgate sensors can measure magnetic field in one [6] or two directions [7]. As a result, printed circuit board based fluxgate sensors may be produced on the mass scale due to the fact that magnetic cores may be easily etched in photolithographic process, whereas sensor body may be produced using standard printed circuit boards production procedures.

On the other hand, as it was previously indicated, printed circuit board (PCB) based fluxgate sensors exhibit sensitivity much lower than bulk magnetic sensors [6]. Moreover, noise to signal ratio in such sensors is much higher than, in e.g., traditional fluxgate sensors utilizing rods made of permalloy or supermalloy [7]. As a result, recent developments of printed circuit board fluxgate sensors is mostly focused on increasing their sensitivity, as well as signal-to-noise ratio in output signal.

\footnotetext{
*corresponding author; e-mail: roman.szewczyk@pw.edu.pl
}

One of the ideas for increasing sensitivity of fluxgate sensor is to add a magnetic flux concentrator to its core [8]. The idea of magnetic flux concentrator is known from other magnetic sensors [9]. However, the efficiency of such concentrator in the case of thin layer fluxgate sensor was not verified previously. This paper aims to fill this gap in the state of the art. Efficiency of magnetic flux concentrator attached to the core of fluxgate sensor was verified on the basis of the finite elements method (FEM) implemented in open-source software.

\section{Principles of operation of fluxgate sensor}

For its operation fluxgate sensor utilizes nonlinearity of magnetizing curve of soft magnetic material. The schematic block diagram of fluxgate sensor is presented in Fig. 1.

External magnetic field $H_{x}$ magnetizes the core in $x$ direction. The internal magnetic field in the core $H_{i}$ is equal [10]:

$$
H_{i}=H_{x}-d M,
$$

where $d$ is demagnetization factor and $M$ is magnetization of the core's material. During the operation of fluxgate sensor, magnetizing windings generate magnetizing field $H_{m}$ in directions opposite to each other.

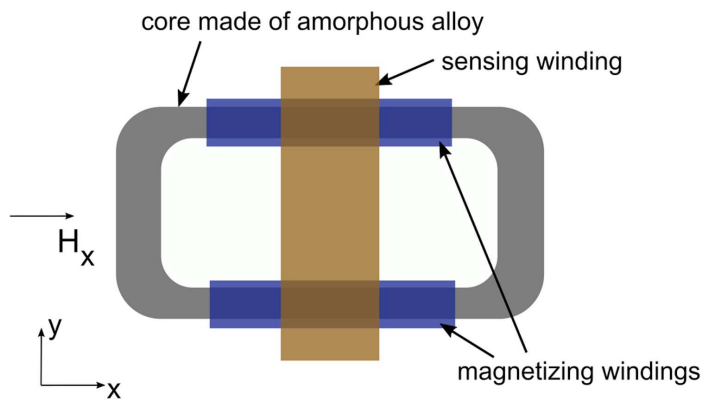

Fig. 1. Schematic block diagram of fluxgate sensor. 
Due to the asymmetry of magnetization (caused by magnetizing field $H_{i}$ ), sensing winding detects even harmonics of magnetizing field frequency. Finally, the amplitude of second harmonics in the output voltage $U_{2 f}$ observed on the sensing winding may be estimated as [11]:

$$
U_{2 f}\left(H_{x}\right)=k n_{s} \mu_{r} H_{x},
$$

where $k$ is the parameter describing the demagnetization factor $d$ and the geometry of fluxgate sensor $n_{s}$ is the number of turns of sensing winding, where $\mu_{r}$ is relative permeability of the sensing core. It is expected that magnetic concentrator will have significant influence on parameter $k$ due to the increase of value of $H_{i}$ for given measured field $H_{x}$.

\section{The method of modelling}

The process of magnetization of a thin layer core of fluxgate sensor was modelled using finite elements method implemented in open-source software. As it was previously indicated [12], finite elements based modelling of thin layers leads to radical increase of the number of elements. For this reason high power desktop computer operating under Linux Mint system was used for the implementation of the models.

The tetrahedral mesh was generated using NETGEN 5.3 open-source meshing program utilizing Delunay method [13]. The maximal height of the element of the core was determined as $100 \mu \mathrm{m}$, whereas the core material thickness was $35 \mu \mathrm{m}$. In spite of the fact that recommended proportions of tetrahedral elements of the core were exceeded, this assumption should efficiently reduce numerical errors.

Finite elements modelling was carried out using ELMER FEM software. The Whitney form of magnetic equations was solved using iterative biconjugate gradient stabilized method [14]. Finally the results of modelling were presented using PARAVIEW visualization software [15], whereas quantitative results were filtered and analyzed using OCTAVE - the open-source alternative of MATLAB.

It should be highlighted that due to the use of opensource software, this method may be applied for both scientific research, as well as for commercial applications connected with introduction of the results of sensor optimization to the market.

\section{Results of modelling}

For the investigation three shapes of cores were considered, as it is presented in Fig. 2. Core presented in Fig. 2a is a typical core of fluxgate sensor. Figure 2b presents core with extended length, which should lead to significant reduction of demagnetization factor $d$. Finally, Fig. 2c presents the amorphous ribbon core with magnetic field concentrator, similar to the solution presented in [8]. Relative magnetic permeability $\mu_{r}$ of all cores was equal to $10^{4}$, whereas core thickness was $35 \mu \mathrm{m}$. Such values are typical for amorphous alloy ribbons in

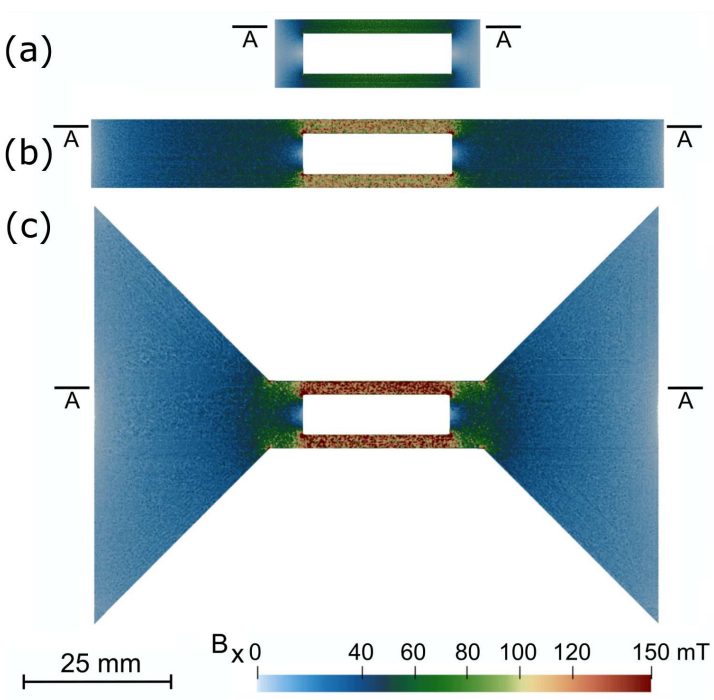

Fig. 2. The distribution of the value of $x$-component of flux density $B$ in cores of fluxgate sensor magnetized by the field $H_{x}=40 \mathrm{~A} / \mathrm{m}$ : (a) typical core of fluxgate sensor produced as a printed board circuit, (b) longer core for demagnetization reduction, (c) core with magnetic field concentrator.
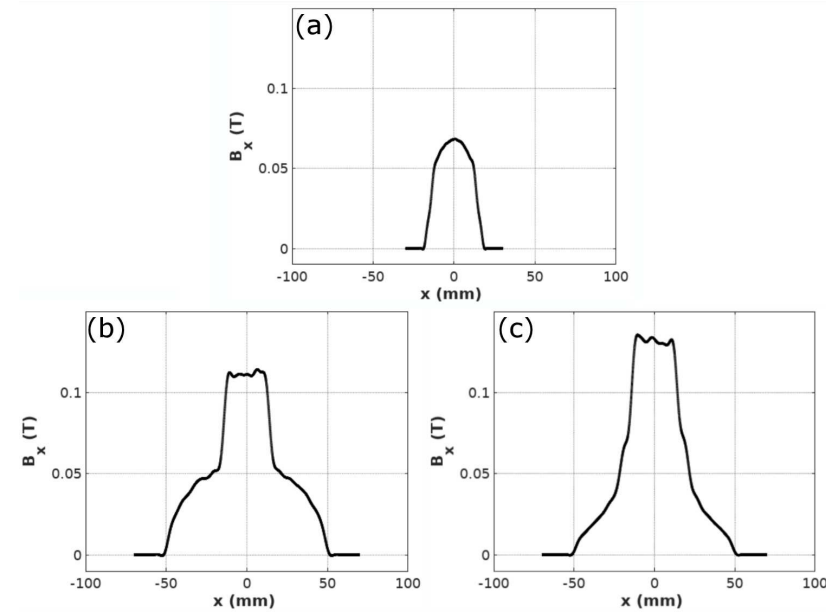

Fig. 3. The distribution of the value of $x$-component of flux density $B$ in the A-A cross-section (Fig. 2) of the cores of fluxgate sensor magnetized by the field $H_{x}=40 \mathrm{~A} / \mathrm{m}$ : (a) typical core of fluxgate sensor produced as a printed board circuit, (b) longer core for demagnetization reduction, (c) core with magnetic field concentrator.

as-quenched state, commonly used as cores for fluxgate sensors produced with printed circuit board technology. All cores were magnetized by constant magnetic field $H_{x}$ equal to $40 \mathrm{~A} / \mathrm{m}$. Figure 2 presents only $x$-component of flux density $B$ in the core due to the fact that only this component is detected by the sensing winding.

As it can be observed in Fig. 2, the quality of convergence during the finite element modelling is moderate. 
This implies that a large number of tetrahedral elements are required for modelling of thin layer. The results of flux density $B_{x}$ distribution in $\mathrm{A}-\mathrm{A}$ cross-section of all cores, after application of the Butterworth digital filter implemented in OCTAVE, are presented in Fig. 3. It can be observed that for typical core of thin layer fluxgate, a value of flux density in the sensing part of the core is non-uniform and does not exceed $70 \mathrm{mT}$. However, for longer core (Figs. 2b and $3 \mathrm{~b}$ ), flux density $B_{x}$ is much higher and reaches the value of about $115 \mathrm{mT}$. This increase is connected to the decrease in demagnetization factor. On the other hand, the use of magnetic concentrator does not cause significant changes in flux density $B_{x}$ distribution. For the third core (Figs. 2c and 3c), flux density $B_{x}$ does not exceed $135 \mathrm{mT}$.

\section{Conclusions}

Our results confirm, that finite elements method may be used for modelling of the thin-layer cores of magnetic sensors. However, previously reported problems with numbers of tetrahedral meshing elements and convergence of models were confirmed.

The results indicate that the increase in the length of the core significantly reduces the demagnetization factor $d$, which increases the value of magnetizing field $H_{i}$ in the core, and leads to significant increase in the sensitivity of fluxgate sensor. In our case this increase exceeded $35 \%$. However, the use of magnetic flux concentrator does not lead to significant increase in sensitivity of fluxgate sensor with core made of thin layer magnetic material. As a result, the use of magnetic flux concentrator in thin layer sensor is not recommended as necessary, and leads to unwanted increase of sensor's dimensions.

\section{Acknowledgments}

This research was partially funded by the statutory funds of the Institute of Metrology and Biomedical Engineering, Warsaw University of Technology.

\section{References}

[1] F. Primdahl, J. Phys. E Sci. Instrum. 12, 241 (1979).

[2] M. Mandea, M. Korte, Geomagnetic Observations and Models, Springer, 2011.

[3] T. Herbich, Archeosciences 33, 77 (2009).

[4] G. Musmann, Y. Afanassiev, Fluxgate Magnetometers for Space Research, Herstellung und Verlag, 2010.

[5] J. Kubik, L. Pavel, P. Ripka, Sens. Actuat. A 130 , 184 (2006).

[6] J. Kubik, P. Ripka, Sens. Actuat. A 143, 237 (2008).

[7] P. Frydrych, R. Szewczyk, J. Salach, K. Trzcinka, IEEE Trans. Magn. 48, 1485 (2012).

[8] R. Rybalko, J. Haueisen, C. Hofmann, Curr. Direct. Biomed. Eng. 1, 22 (2015).

[9] J. Valadeiro, S. Cardoso, R. Macedo, A. Guedes, J. Gaspar, P.P. Freitas, Micromachines 7, 88 (2016).

[10] D.C. Jiles, Introduction to Magnetism and Magnetic Materials, Chapman and Hall, 1998.

[11] P. Ripka, Magnetic Sensors and Magnetometers, Artech 2001.

[12] R. Szewczyk, in: 22th Int. Conf. on Applied Physics of Condensed Matter, Strbské Pleso (Slovakia), 2016.

[13] J. Schöberl, NGSolve library.

[14] CSC - IT Center for Science, Elmer.

[15] Kitware, ParaView. 